\title{
$X$-ray variations in the inner accretion flow of dwarf novae
}

\author{
Ş. Balman ${ }^{1}$ and M. Revnivtsev ${ }^{2}$ \\ 1 Middle East Technical University, Dept. of Physics, Inönü Bulvarı, 06531, Ankara, Turkey \\ e-mail: solen@astroa.physics.metu.edu.tr \\ 2 Space Research Institute, Russian Academy of Sciences, Profsoyuznaya 84/32, 117997 Moscow, Russia \\ Received 24 April 2012 / Accepted 28 August 2012
}

\begin{abstract}
Aims. We study the inner disk structure of dwarf novae (DNe; i.e., nonmagnetic cataclysmic variables).

Methods. We performed power spectral analysis of the X-ray light curves obtained using the Rossi X-ray Timing Explorer (RXTE) and X-ray Multi-mirror Mission (XMM-Newton) data. We fit the power spectra with a simple model that describes variability as a result of matter fluctuations. In addition, we applied cross-correlation analysis of simultaneous UV and X-ray light curves using the $X M M$-Newton data to determine time lags between the different wavelength data.

Results. For five DN systems, SS Cyg, VW Hyi, RU Peg, WW Cet, and T Leo we show that the UV and X-ray power spectra of their time variable light curves are similar in quiescence. All of them show a break in their power spectra, which in the framework of the model of propagating fluctuations indicates inner disk truncation. We derive the inner disk radii for these systems in a range of $(10-3) \times 10^{9} \mathrm{~cm}$. We analyze the RXTE data of SS Cyg in outburst and compare it with the power spectra, obtained during the period of quiescence. We show that during the outburst the disk moves towards the white dwarf and recedes as the outburst declines. We calculate the correlation between the simultaneous UV and X-ray light curves of the five DN studied in this work, using the XMMNewton data obtained in the quiescence and find X-ray time lags of 96-181 s. This can be explained by the travel time of matter from a truncated inner disk to the white dwarf surface.

Conclusions. We suggest that, in general, DN may have truncated accretion disks in quiescence, which can also explain the UV and X-ray delays in the outburst stage and that the accretion may occur through coronal flows in the disk (e.g., rotating accretion disk coronae). Within the framework of the model of propagating fluctuations, the comparison of the X-ray/UV time lags observed by us in the case of DN systems with those detected for a magnetic intermediate polar allows us to make a rough estimate of the viscosity parameter $\alpha \sim 0.25$ in the innermost parts of the accretion flow of DN systems.
\end{abstract}

Key words. accretion, accretion disks - methods: data analysis - binaries: close - stars: dwarf novae - X-rays: stars

\section{Introduction}

Cataclysmic variables (CVs) are interacting binaries hosting a white dwarf (WD) primary star accreting material from a latetype main sequence star. Accreting material forms a disk that is expected to reach all the way to the WD in cases where the magnetic field of the WD is weak $(B<0.01 \mathrm{MG})$, and these systems are referred to as nonmagnetic CVs (see Warner 1995, for a review)

In our work we consider dwarf novae (DNe), a subclass of nonmagnetic cataclysmic variables. In such systems, the matter which is transferred from the secondary star to the Roche lobe of the primary, does not form a stationary flow to the WD surface, but the mass transfer rate is diminishing towards the WD during the so-called quiescent state. These states are interrupted every few weeks to tens of years by the enhanced accretion flow (outbursts) that lasts days to weeks, and the systems significantly brighten (bolometrically).

The material in the inner disk of DNe initially moving with the Keplerian velocity dissipates its kinetic energy in order to accrete onto the slowly rotating WD, creating a boundary layer (Lynden-Bell \& Pringle 1974; Kippenhahn \& Thomas 1978; Narayan \& Popham 1993; Godon et al. 1995). Observations show that during the quiescence (low-mass accretion rates in the innermost parts of the flow) a significant or dominant fraction of the bolometric emission of $\mathrm{DNe}$ is emitted via the bremsstrahlung process from an optically thin hot plasma in the hard X-rays (Patterson \& Raymond 1985; Narayan \& Popham 1993). Typical characteristic of the quiescent X-ray emission is a multitemperature quasi-isobaric cooling flow model of plasma emission with temperatures of $6-50 \mathrm{keV}$ and an accretion rate of $10^{-12}-10^{-10} M_{\odot} / \mathrm{yr}$ with $L_{\mathrm{x}}<10^{33} \mathrm{erg} \mathrm{s}^{-1}$ (Perna et al. 2003; Pandel et al. 2005; Kuulkers et al. 2006; Rana et al. 2006; Balman et al. 2011).

The observational appearance of quiescent DNe indicates that a significant part of the energy release during ongoing accretion is happening in the optically thin region near the WD. Thus the accretion flow is likely changing its character from optically thick disk-like flow in the its outer parts to an optically thin "corona"-like flow close to the WD.

This truncation of the optically thick accretion disk in DNe in quiescence was observationally invoked due to the observed time lags between the optical and UV fluxes in the rise phase of the outbursts (see reviews Lasota 2001, 2004) or due to unusual shape of the optical spectra or light curves of DNe (see e.g. Linnell et al. 2005; Kuulkers et al. 2011). Theoretical support for such a two-phase flow was given by a model of the disk evaporation of Meyer \& Meyer-Hofmeister (1994). This model was later elaborated to show that the disk evaporation (coronal "syphon" flow) may create optically thick-optically thin transition regions at various distances from the WD (Liu et al. 1997; Mineshige et al. 1998). Attempts to obtain a map of the accretion disk in cataclysmic variables were done with the help of 
Table 1. Log of observations (List of observation IDs) used in our work.

\begin{tabular}{ll}
\hline \hline RXTE & 20033013100-20033014200, 50011018000-50011019700, 50012010101-50012010109 \\
Quiescence & \\
RXTE & $40012010200,40012010500,40012010600,50011013100-50011014300,95421010100,95421010200$, \\
X-ray dips & $95421010800,95421011000,954210200-954210204$ \\
RXTE & $40012011100-40012011800,50011015800-50011017100,95421010300-95421010301$, \\
X-ray Peak & $50012010104-50012010108$ \\
XMM-Newton & 0111310201 (SS Cyg), 0111970301 (VW Hyi), 0111970901 (WW Cet), 0551920101 (RU Peg), \\
Quiescence & 0111970701 (T Leo) \\
\hline
\end{tabular}

Notes. All RXTE data is of SS Cyg.

the eclipse-mapping method (e.g. Horne 1985), but this method has its own limitations and is not very sensitive to the innermost parts of the accretion disk.

Recently, an additional diagnostic tool has been proposed the aperiodic variability of brightness of sources. Virtually all accreting sources demonstrate aperiodic variability of their brightness on a wide range of time scales (see Bruch 1992, for a review of aperiodic variability of cataclysmic variables). While the long time-scale variability might be created in the outer parts of the accretion disk (see e.g. Warner \& Nather 1971), the relatively fast time variability (at $f>$ few $\mathrm{mHz}$ ) originates in the inner parts of the accretion flow (see e.g. Bruch 2000; Baptista \& Bortoletto 2004).

Properties of this noise is similar to that of the X-ray binaries with neutron stars and black holes. These properties are quite peculiar, which does not allow it to be explained as a sum of independent burst-like events in the region of main energy release. In particular, the variability demonstrates a very tight relation between the rms amplitude of variations and the average flux level (see e.g. Uttley \& McHardy 2001; Scaringi et al. 2012). The variability spans an extremely wide range of frequencies with the same power-law slopes (see e.g. Churazov et al. 2001). Now, the widely accepted model of origin for this aperiodic flicker noise is a model of propagating fluctuations (Lyubarskii 1997; Churazov et al. 2001; Uttley \& McHardy 2001; Arévalo \& Uttley 2006; Revnivtsev et al. 2009, 2010; Uttley et al. 2011).

In the framework of this model, the modulations of the light are created by variations in the instantaneous value of the mass accretion rate in the region of the energy release. These variations in the mass accretion rate, in turn, are inserted into the flow at all radii of the accretion disk due to the stochastic nature of its viscosity and then transferred toward the compact object. Fast variations in the mass accretion rate inserted into the flow at distances closer to the central object modulate the mass flow coming into these regions from outer parts of the accretion disk.

This model predicts that the truncated accretion disk should lack some part of its variability at high Fourier frequencies, i.e. on the time scales shorter than a typical time scale of variability at the inner edge of the disk. This prediction was checked for the accreting systems, in which the disks are indeed truncated owing to the interaction with the compact object magnetospheres, in particular, accreting magnetic neutron stars (Revnivtsev et al. 2009) and accreting magnetic WDs (Revnivtsev et al. 2010). The breaks revealed in the power spectra of these accreting binaries allowed one to estimate of the inner radius of the accretion disk. In particular, in the work of Revnivtsev et al. (2011), it was shown that the inner truncation radius of the accretion disk in EX Hya estimated from the variability arguments was quite compatible with those estimated with the help of completely different physical effects.

In this paper we would like to apply the similar diagnostic tool to estimate the inner boundary of the optically thick accretion disk in nonmagnetic WDs - dwarf novae. We used $X M M-N e w t o n$ and RXTE data to study the broad-band noise in DN and to calculate the inner disk radii for five systems, SS Cyg, VW Hyi, RU Peg, WW Cet, and T Leo. Basing on not detecting any periodic X-ray light variations (with the very tight upper limit) in the flux of the enlisted systems, we assume that they contain nonmagnetic WDs, while such a classification for SS Cyg is challenged by some authors (e.g. Lombardi et al. 1987; Giovannelli \& Sabau-Graziati 1999). This is not particularly important for here because we study the accretion disk truncation, and the origin of this truncation might have different nature in different sources.

We infer from our results that these systems have truncated disks at large radii and that some form of rotating coronal flow (e.g., ADAF disks and/or accretion disk coronae) exists in DN systems where the material is transported to the surface of the WD.

\section{Data and observation}

The XMM-Newton Observatory (Jansen et al. 2001) has three $1500 \mathrm{~cm}^{2}$ X-ray telescopes each with an European Photon Imaging Camera (EPIC) at the focus. Two of them have a multiobject spectrometer (MOS) CCDs (Turner et al. 2001) and the last one uses pn CCDs (Strüder et al. 2001) for data recording. The optical monitor (OM), a photon-counting instrument, is a co-aligned $30-\mathrm{cm}$ optical/UV telescope, providing the first possibility of observing from a single platform simultaneously in the X-ray and optical/UV regime (Mason et al. 2001). For our timing purposes we utilized the data collected with the EPIC pn cameras in the partial or full window imaging mode, and the OM data using the fast imaging mode ( $\geq 0.5 \mathrm{~s}$ time resolution) with the UVW1 filter (240-340 nm). The time resolution of imaging modes are $70 \mathrm{~ms}$, the timing mode observations have $30 \mu \mathrm{s}$ resolution and the burst mode goes down to $7 \mu$ s in time resolution. We summarize the archival XMM-Newton data used in this analysis on Table 1. We utilized the EPIC pn data and the MOS data, when necessary for our analysis, since EPIC has the adequate sensitivity and timing resolution for our study. A medium optical blocking filter was used with all the EPIC cameras. We analyzed the pipeline-processed data using Science Analysis Software (SAS) version 11.0.0. Data (single- and double-pixel events, i.e., patterns $0-4$ with Flag $=0$ option) were extracted from a circular region of radius $30^{\prime \prime}$ for pn, (MOS1 and MOS2) in order to perform temporal analysis, together with the background events extracted from a source free zone normalized to the source extraction area. We checked/cleaned the pipeline-processed events file from the existing flaring episodes during the analysis for all the objects. The OM data was analyzed using the OMFCHAIN with $1 \mathrm{~s}$ time resolution.

We used Rossi X-ray Timing Explorer (RXTE; Bradt et al. 1993) archival data for the analysis of the outburst data of SS Cyg and the quiescence data for comparison. The data 
Ş. Balman and M. Revnivtsev: X-ray variations in the accretion flow of dwarf novae

Table 2. Break frequencies, disk truncation radii, and time delays of the dwarf novae analyzed in this work.

\begin{tabular}{lccccc}
\hline \hline Source & State & Break Freq. $(\mathrm{mHz})$ & Radius $\left(\times 10^{9} \mathrm{~cm}\right)$ & Delay $(\mathrm{s})$ & $\log \left(L_{U V W 1} / L_{X}\right)$ \\
\hline SS Cyg & (quiescence-XMM) & $5.6 \pm 1.4$ & $4.8 \pm 1.2$ & $166-181\left(\chi_{v}^{2}=0.8\right)$ & -4.3 \\
SS Cyg & (quiescence-RXTE) & $4.5 \pm 1.3$ & $5.5 \pm 1.8$ & & \\
SS Cyg & (X-ray Dips) & $50.0 \pm 20.0$ & $1.1 \pm 0.5$ & & \\
SS Cyg & (X-ray peak) & $9.7 \pm 1.5$ & $3.3 \pm 0.5$ & & \\
RU Peg & (quiescence) & $2.8 \pm 0.5$ & $8.2 \pm 1.5$ & $97-109\left(\chi_{v}^{2}=1.7\right)$ & -3.1 \\
VW Hyi & (quiescence) & $2.0 \pm 0.6$ & $8.1 \pm 2.5$ & $103-165\left(\chi_{v}^{2}=0.5\right)$ & -2.7 \\
WW Cet & (quiescence) & $3.0 \pm 1.7$ & $6.8 \pm 3.8$ & $118-136\left(\chi_{v}^{2}=1.6\right)$ & -3.6 \\
T Leo & (quiescence) & $4.5 \pm 1.5$ & $4.0 \pm 1.3$ & $96-121\left(\chi_{v}^{2}=1.4\right)$ & -3.7 \\
\hline
\end{tabular}

Notes. The last column is the logarithm of the ratio between the UV and X-ray luminosities. The UV luminosities are in erg s ${ }^{-1} \AA^{-1}$ obtained using the XMM-Newton OM UVW1 filter. The errors represent $90 \%$ confidence level. The $\chi_{v}^{2}$ values are for the delays calculated from fits with the subtracted CCFs except for SS Cyg for which a single Lorentzian fit was applied.

were obtained by the Proportional Counter Array (PCA; Jahoda et al. 2006) instrument aboard RXTE. The PCA consists of five xenon-filled detector units (PCUs) with a total effective area of $6200 \mathrm{~cm}^{2}$ at $5 \mathrm{keV}$. It is sensitive in the range $2-60 \mathrm{keV}$, the energy resolution is $17 \%$ at $5 \mathrm{keV}$, and the time resolution capability is $1 \mu \mathrm{s}$. A log of observations can be found in Table 1 . RXTE/PCA background was estimated with the help of the model appropriate for faint sources. Light curves of the source were extracted with the standard procedures (i.e., SAEXTRCT) from the data of 1-3 PCUs mainly in the entire PCA energy band utilizing the "Standard 1" data with a $0.125 \mathrm{~s}$ time resolution. As an additional check of the results, we analyzed the Good Xenon mode data in the energy band 3-20 keV and obtained similar results. All event arrival times were corrected to the solar system barycenter.

All light curves were background-subtracted for the analysis. The data and light curves were analyzed using HEAsOFT version V6.9.

\section{Analysis and results}

\subsection{SS Cyg}

We prepared light curves using the standard procedures as described in the previous section. To measure the broad-band noise, we derived and averaged several power spectra for each source from the calculated light curves. The power spectral densities (PDS) expressed were calculated in terms of the fractional rms amplitude squared following from Miyamoto et al. (1991),

$P_{j}=2\left|A_{j}\right|^{2} / N_{\mathrm{ph}} C \quad A_{j}=\sum x_{n} \mathrm{e}^{\mathrm{i} \omega_{n} t_{n}}$.

In this prescription, $t_{n}$ is the time label for each time bin, $x_{n}$ is the number of counts in these bins, $N_{\text {ph }}$ the total number of photons in each light curve, and $\mathrm{C}$ the average count rate in each time segment used to construct PSD. The white noise levels were subtracted hence leaving us with the rms fractional variability of the time series in units of $(\mathrm{rms} / \mathrm{mean})^{2} / \mathrm{Hz}$. Next, we multiplied the rms fractional variability per hertz with the frequencies at which they were calculated to yield an rms fractional variability squared, thus our PDS are $v P_{v}$ versus $v$. For the model fitting we used a simple analytical model

$P(v) \propto v^{-1}\left(1+\left(\frac{v}{v_{0}}\right)^{4}\right)^{-1 / 4}$,

which was proposed to describe the power spectra of sources with truncated accretion disks (see e.g. Revnivtsev et al. 2010, 2011).
We used the archival RXTE data of SS Cyg in quiescence and outburst listed in Table 1 to derive the broad-band noise of the source in different states (i.e., accretion rates). For the outburst phases, we investigated times during the X-ray suppression (e.g., the X-ray dips; optical peak phases of the outburst) and the X-ray peak. This, in turn is expected to show the motion of the flow in the inner regions of the disk and the geometry of the inner disk. For details of the source spectra and light curves during outburst and quiescence (see McGowan et al. 2004). Figure 1 shows a compilation of the PDS of SS Cyg obtained from RXTE data. This includes the RXTE PDS of the quiescent phase data, the RXTE PDS during the optical bright phase (i.e., X-ray dips), and the PDS during the X-ray peak of the outburst.

The source PDS from the RXTE data are fitted with the described model in the above paragraph; $v P_{v}=\mathrm{P} 2 \times\left(1+\left(\frac{v}{\mathrm{P} 1}\right)^{4}\right)^{-1 / 4}$, which uses two parameters P1 the break frequency noted as $v_{0}$ in the analytical model, and P2, the normalization that determines the power level of the flat part of the broken powerlaw. The reduced $\chi^{2}$ values of the fits are $0.62,1.5$, and 0.4 for the quiescence, the X-ray peak, and the X-ray dips, respectively. The resulting break frequencies are displayed in Table 2, and the corresponding disk truncation radii are calculted and displayed in the same table using the simple relation $v_{0} \simeq\left(\mathrm{GM}_{\mathrm{WD}} / R_{\mathrm{in}}^{3}\right)^{1 / 2} / 2 \pi$ (assuming $1 M_{\odot}$ for the WD mass). The broad-band noise structure of the Keplerian disks often show $\propto f^{-1 \cdots-1.3}$ dependence on frequency (Churazov et al. 2001; Gilfanov \& Arefiev 2005), and this noise will show a break if the optically thick disk truncates as the Keplerian motion subsides. We detect that all RXTE PDS show breaks, and this implies that the disk in SS Cyg is truncated at large distances compared with the size of the WD.

As clearly shown in Table 2, we detect that the inner edge of the disk is farther out during the quiescence, and the disk moves inwards to about $1.1 \times 10^{9} \mathrm{~cm}$ during the peak of the optical outburst and starts moving out to about $3.3 \times 10^{9} \mathrm{~cm}$ during the $\mathrm{X}$-ray peak of outburst. It finally reaches about $5.5 \times 10^{9} \mathrm{~cm}$ at the quiescent flux levels after the outburst has subsided.

The quiescent XMM-Newton data of SS Cyg obtained at a different date is used to validate the quiescent RXTE PDS and check the disk truncation radius. The two fitted quiescent PDS are very similar as displayed in Fig. 2. The resulting break frequency and the disk truncation radius are given in Table 2 . The reduced $\chi^{2}$ of the fit using the XMM-Newton (EPIC pn) PDS is 1.4. In addition, we calculated the PDS in the UV from the OM data (XMM-Newton) and included it in Fig. 3 for comparison. The break frequency and the disk truncation radius calculated from the UV overlap with the X-ray results within the $95 \%$ confidence level error ranges. The reduced $\chi^{2}$ of the fit to the XMM-Newton (OM) PDS is 0.4 . 

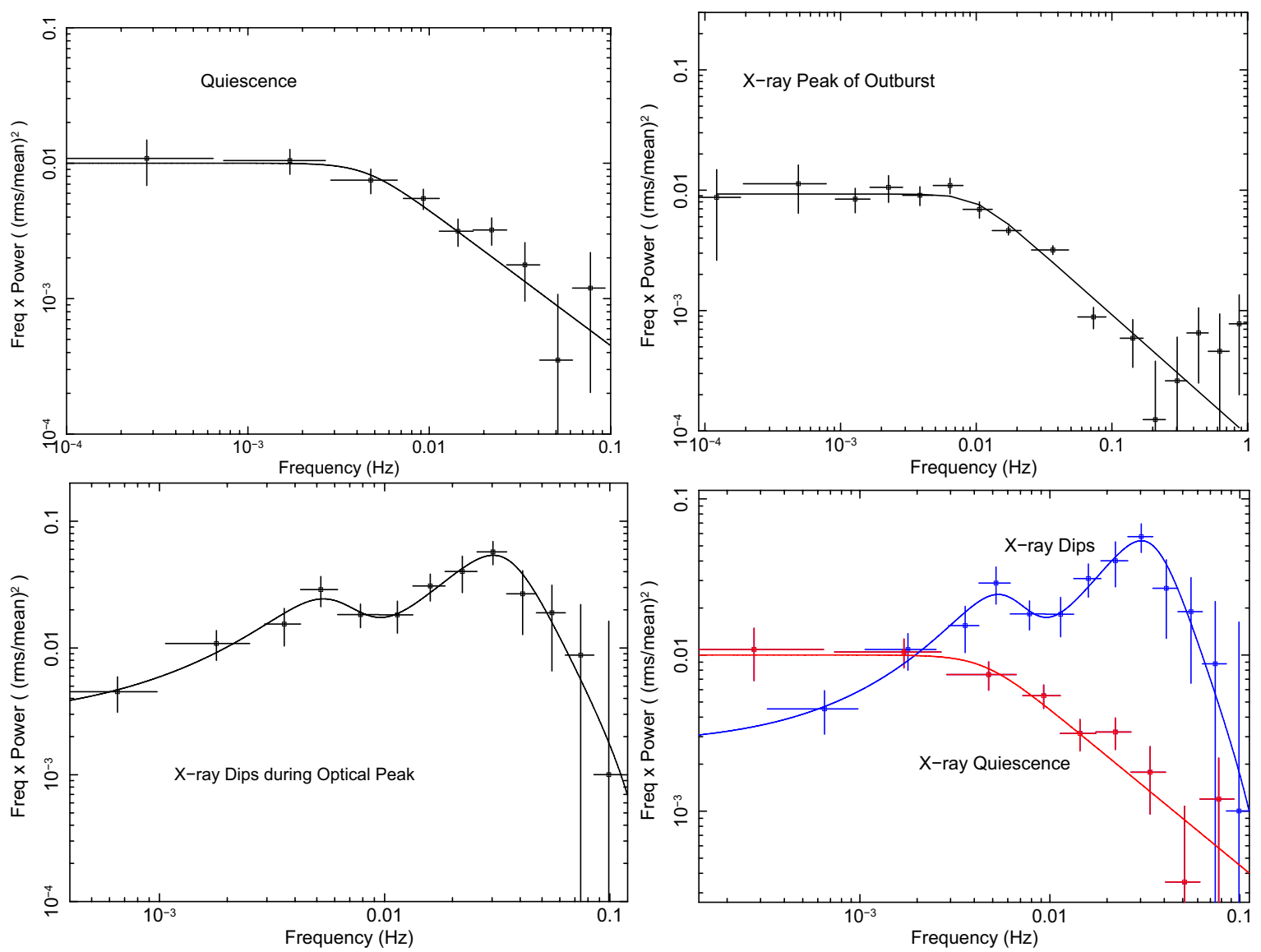

Fig. 1. Power spectra of SS Cyg in outburst obtained from a collection of archival RXTE data listed in Table 1. The PDS at different times are ordered as the quiesence on the top lefthand side, the X-ray peak on the top righthand side, and the X-ray dips during the optical peak on the bottom lefthand side. On the bottom righthand side, the PDS of SS Cyg in quiescence and during the X-ray suppression (optical peak) is shown for comparison. The solid lines show the fit with the propagating fluctuations model for the top figures and for the PDS of the X-ray dips two Lorentzians along with the propagating fluctuations model are used to achieve the best fitting results. The reduced $\chi^{2}$ values of the fits are 0.62 , 1.5 , and 0.4 for the quiescence, the X-ray peak and the X-ray dips, respectively.

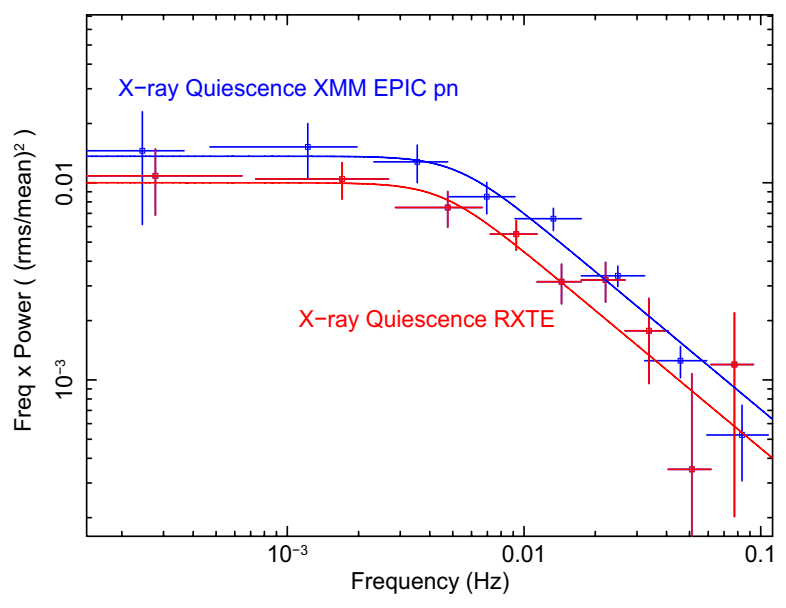

Fig. 2. X-ray power spectra of SS Cyg in quiescence obtained from the XMM-Newton EPIC pn data. Dark blue data points are for the $X M M-N e w t o n$ EPIC pn PDS and the overlayed red data points are for the RXTE PDS. The solid lines are the fitted curves using the propagating fluctuations model. The reduced $\chi^{2}$ of the fit to the XMM-Newton (EPIC pn) PDS is 1.4.

\subsection{Other dwarf novae}

To check if the truncation of the inner disk radius is unique to SS Cyg, we used the archival XMM-Newton data of other

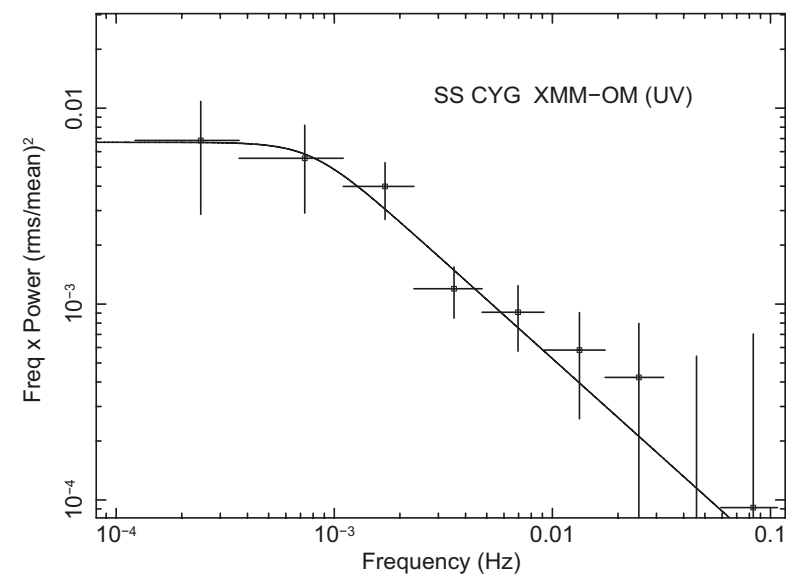

Fig. 3. UV power spectrum of SS Cyg in quiescence obtained from the XMM-Newton OM data. Black solid line is the fitted curve using the propagating fluctuations model. The reduced $\chi^{2}$ of the fit to the XMM-Newton (OM) PDS is 0.4 .

different kinds of DNe. We prepared light curves in the same manner and prepared PDS from simultaneous X-ray (EPIC) and UV (OM) data. Figures 4-9 show the PDS of four other DN; RU Peg (U Gem type), VW Hyi (SU UMa type), WW Cet (Z Cam type), and T Leo (SU UMa type). We omitted the UV PDS of WW Cet and T Leo as a result of low statistical 
Ş. Balman and M. Revnivtsev: X-ray variations in the accretion flow of dwarf novae

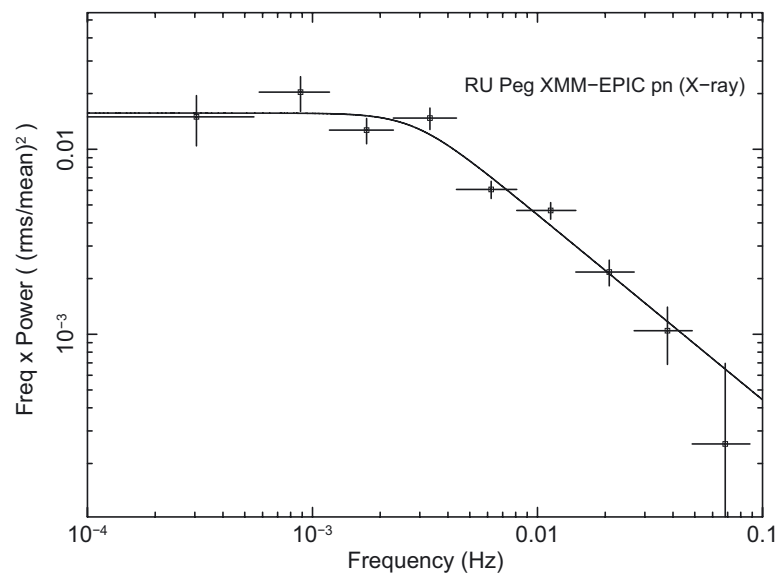

Fig. 4. X-ray power spectrum of RU Peg in quiescence obtained from the XMM-Newton EPIC pn data. Black solid line is the fitted curve using the propagating fluctuations model. The reduced $\chi^{2}$ of the fit to the $X M M$-Newton (EPIC pn) PDS is 1.5 .

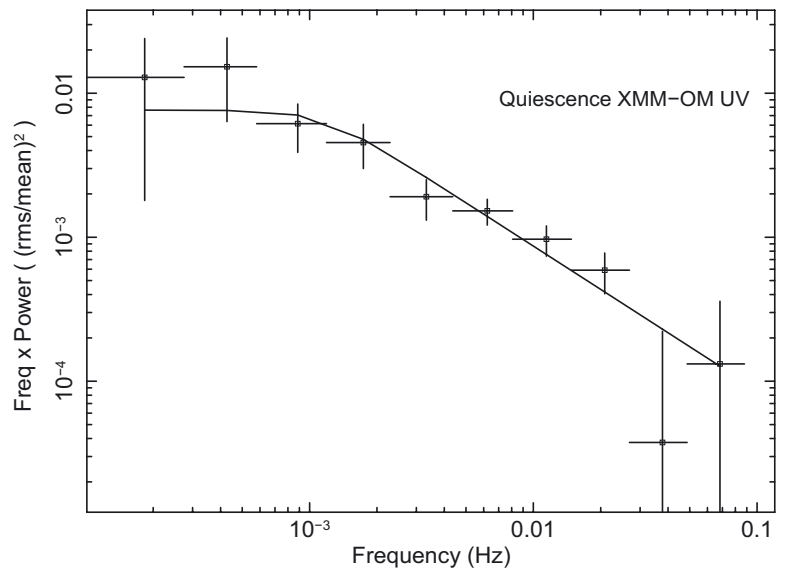

Fig. 5. UV power spectrum of RU Peg in quiescence obtained from the XMM-Newton OM data. Black solid line is the fitted curve using the propagating fluctuations model. The reduced $\chi^{2}$ of the fit to the XMM-Newton (OM) PDS is 0.8 .

quality. We applied the same fit (using the same functional form) to the PDS of these DNe as was done for SS Cyg. The fits to the various PDS yield reduced $\chi^{2}$ in a range $0.2-1.4$. The particular values are indicated in the figure captions. All PDS indicate disk truncation at radii larger than the size of the WD. We display the break frequencies calculated using the X-ray data and the disk truncation radii in Table 2. The inner disk radii are calculated as was done for SS Cyg, and we assumed WD masses as listed by Pandel et al. (2005). The break frequency and the disk truncation radii calculated from the UV overlap with the X-ray results within the $95 \%$ confidence level error ranges.

The orbital period of VW Hyi was removed from the UV PDS by modeling a narrow Loretzian, and this period is not detected in the X-ray PDS. We underline that our UV data for all five DNe do not have long enough duration to derive the PDS characteristics securely at low frequencies. Thus, we cannot infer if there is any contribution to red noise from other components of flickering noise, such as the accretion hot spot, overflowing accretion stream, and the spiral waves as proposed using optical wavelength data (see e.g. Bruch 2000; Baptista \& Bortoletto 2004).

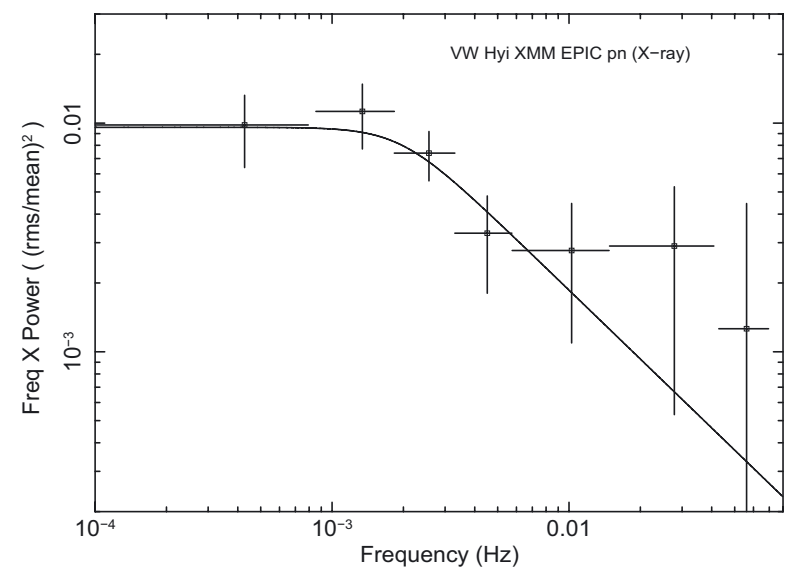

Fig. 6. X-ray power spectrum of VW Hyi in quiescence obtained from the XMM-Newton EPIC data. Overplotted curve is the fitted propagating fluctuations model. The reduced $\chi^{2}$ of the fit to the XMM-Newton (EPIC pn) PDS is 0.5 .

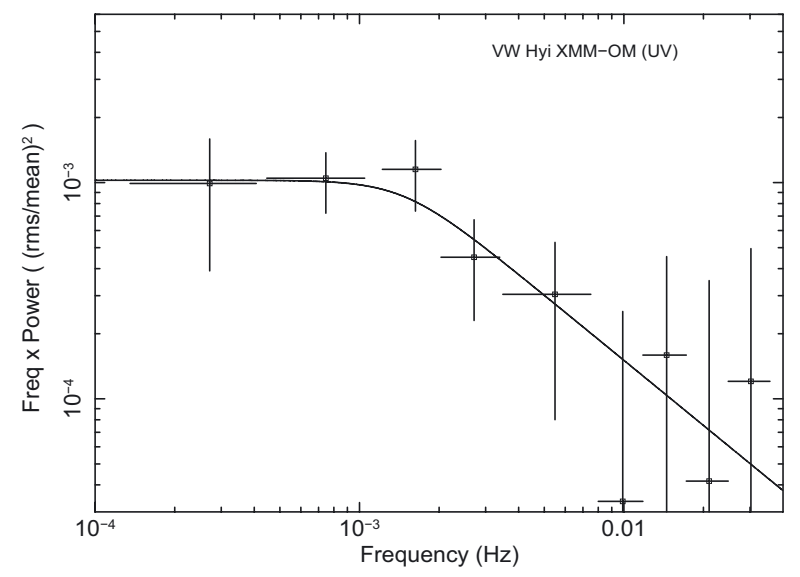

Fig. 7. UV power spectrum of VW Hyi in quiescence obtained from the XMM-Newton OM data. Overplotted curve is the fitted propagating fluctuations model. The reduced $\chi^{2}$ of the fit to the XMM-Newton (OM) PDS is 0.4 .

\subsection{The cross-correlations}

If the model of the propagating fluctuations correctly describes the time variability of the X-ray and optical/UV flux of DNe, then we should expect some particular way to correlate the brightness of systems at these energies. In particular, the opti$\mathrm{cal} / \mathrm{UV}$ light, generated as reprocessing of the X-ray emission from the central regions should lag with respect to the X-rays consistent with the light crossing time (typically less than a second for majority of DNe, but $\sim 5-6 \mathrm{~s}$ for the largest binaries, like RU Peg). But the optical/UV light variations, generated as an energy release of variable mass accretion rate at the inner edge of the accretion disk, should lead the X-ray emission with the time lag equal to the time needed for the matter to travel from the inner edge of the disk to the central regions of the accretion flow in the vicinity of the WD, where the bulk of the X-ray emission is generated.

To study this question, we calculated the cross-correlation between the two simultaneous light curves (X-ray and UV), using the archival XMM-Newton data utilizing HEAsOFT task CROSSCOR. To obtain the CCFs (cross-correlation functions), we divided our datasets into several pieces using $1-5 \mathrm{~s}$ binning in light curves and calculated CCFs for each of them. 


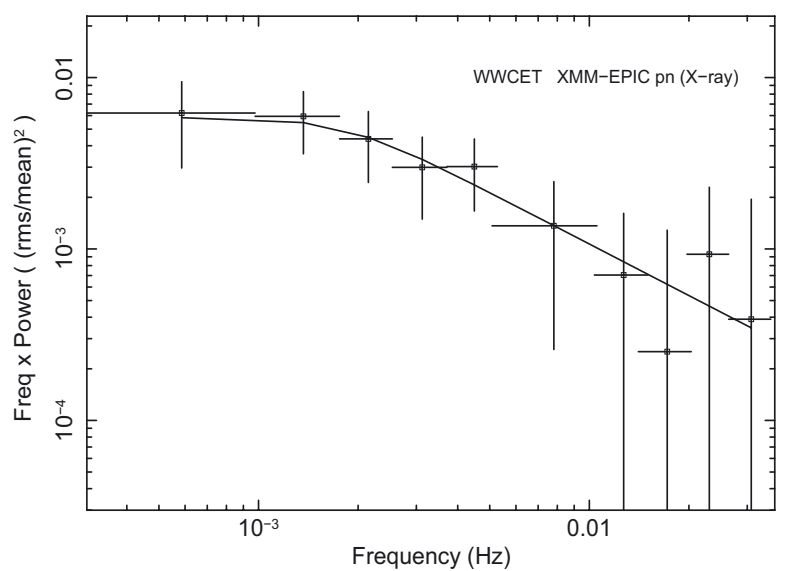

Fig. 8. X-ray power spectrum of WW Cet in quiescence obtained from the XMM-Newton EPIC pn data. Overplotted is the fitted curve using the the propagating fluctuations model. The reduced $\chi^{2}$ of the fit to the XMM-Newton (EPIC pn) PDS is 0.2 .

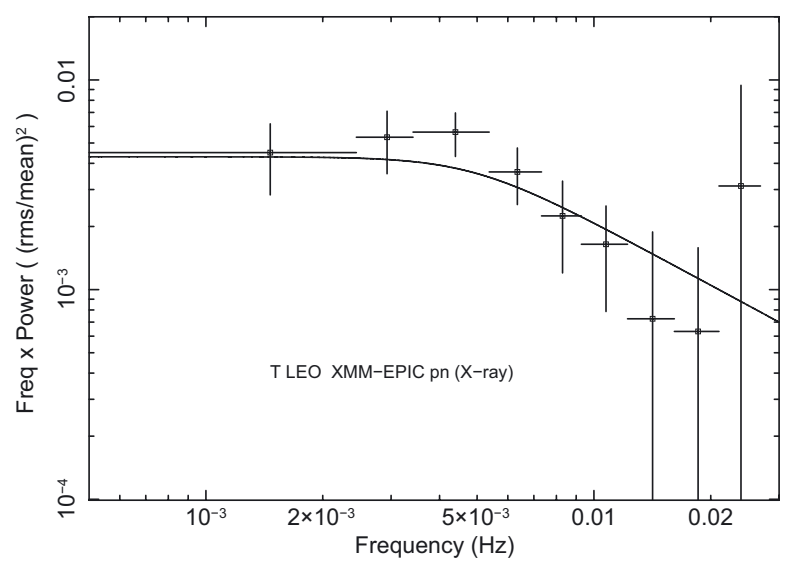

Fig. 9. Power spectrum of $\mathrm{T}$ Leo in quiescence obtained from the $X M M$-Newton EPIC pn data. Overplotted is the fitted curve using the power relation for the noise from the propagating fluctuations model. The reduced $\chi^{2}$ of the fit to the XMM-Newton (EPIC pn) PDS is 0.7 .

The $\mathrm{CCF}$ at each lag $j, \operatorname{CCF}(\mathrm{j})$ is computed with the expression

$$
C C F(j)=\frac{\sum t\left(x_{\mathrm{uv}}(t)-\bar{x}_{\mathrm{uv}}\right)\left(x_{\mathrm{x}-\mathrm{ray}}(t+j)-\bar{x}_{\mathrm{x}-\mathrm{ray}}\right)}{N(j)\left(\sigma_{\mathrm{uv}}^{2} \sigma_{\mathrm{x}-\mathrm{ray}}^{2}\right)^{1 / 2}}
$$

where $j=0, \pm \Delta t \ldots$. The summation goes from $t=\Delta t$ to $(N-j) \Delta t$ for zero and positive $j$ and from $t=(1-j) \Delta t$ to $N \Delta t$ for negative $j$, where $N$ is the total number of points in the light curve while $N(j)$ is the number of pairs that contribute to the calculation of CCF at lag $j$. The resulting correlation coefficients from the above expression as a function of time lag is shown in Fig. 10 for all five $\mathrm{DN}$ we analyzed. We note that this correlation analysis was already presented for RU Peg in Balman et al. (2011) and for VW Hyi in Pandel et al. (2003), but we include them here for comparison and completeness of our analysis. The crosscorrelation coefficient is normalized to have a maximum value of 1 . The error bars are evaluated as root mean square deviations from the average value of the cross-corelation of each measurement (made by using the segments of the whole light curve).

The curves for all the DNe show clear asymmetry indicating that some part of the UV flux is leading the X-ray flux. In addition, we detect a strong peak near zero time-lag for RU Peg, WW Cet and T Leo, suggesting a significant zero-lag correlation
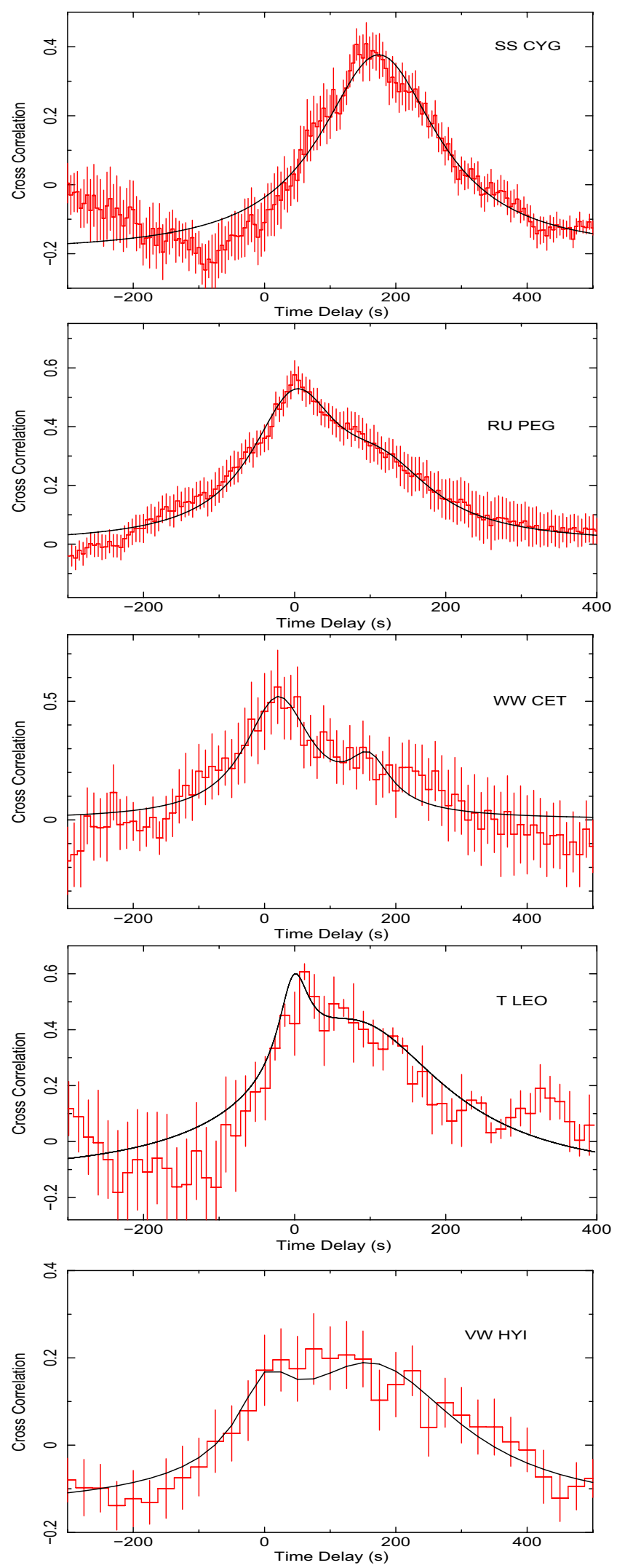

Fig. 10. Cross-correlation of the EPIC pn (X-ray) and OM (UV) light curves with $1 \mathrm{~s}$ time resolution. The CCFs are displayed for SS Cyg, RU Peg, WW Cet, T Leo, and VW Hyi from the top to the bottom of the figure. The correlation coefficient is normalized to a maximum value of 1 . The two-component Lorenzian fits are shown as solid black lines (except for SS Cyg where a single Lorentzian was used). The reduced $\chi^{2}$ values are $0.8,0.4,0.45,1.2$, and 0.45 from the top to the bottom panels of the figure. 
between the X-rays and the UV light curves. Our reanalysis of the cross-correlation for VW Hyi also reveals a correlation at zero time-lag in addition to Pandel et al. (2003) which we include in Fig. 10. The positive time lag, leading to an asymmetric profile for the four DNe above and the shifted profile for SS Cyg show that the X-ray variations are delayed relative to those in the UV.

In order to calculate an average time lag that would produce the asymmetric or shifted profile, we fitted the varying cross-correlation by two Lorentzians with time parameter fixed at $0.0 \mathrm{lag}$ (not assumed for SS Cyg) and the other set as free. A Lorentzian has a functional form $F(y)=\mathrm{P} 3 \times\left(1+\left(\frac{2 \cdot(\mathrm{x}-\mathrm{P} 1)}{\mathrm{P} 2}\right)^{2}\right)^{-1}$ where $\mathrm{P} 1$ is the time parameter that yields the time lag, $\mathrm{P} 2$ the FWHM of the Lorentzian, and P3 the normalization. We only report the parameter P1 for the measure of the time lag and its error. Our PDS are broken power laws, so our ACF/CCF cannot be Gaussian-shaped. In order for the ACF/CCF to be Gaussian, our PDS should also be Gaussian-shaped since the PDS is a cosine transfrom of the ACF (auto correlation function). Our expected $\mathrm{ACF} / \mathrm{CCF}$ should be more extended than a Gaussian where a Lorentzian is, then, an acceptable choice. The resulting fits are displayed in Fig. 11. The delay times vary in a range 70-240 s for the five DN. The fit for RU Peg is taken from Balman et al. (2011). The reduced $\chi^{2}$ of the fits in Fig. 10 are 0.8, 0.4, 0.45, 1.2 , and 0.45 from the top to the bottom panels of the figure.

To double-check these generic fits, we constructed a more physically motivated plot of time-correlation using the shape of the auto-correlation of the X-ray light curves. Assuming that the zero time-lag signal on the original cross-correlation plot is created by a simple transformation of the X-ray variability into the UV light, we subtracted that part adopting the shape of X-ray auto-correlation function. The amplitude of this zero time-lag component was taken to provide smooth behavior of the subtracted cross-correlation function across zero delay. The subtracted cross-correlation functions directly yield the shifted time-lag component. This is applied to the four DNe that show the zero time-lag in their cross-correlation and the residuals are fitted with a single Lorentzian to calculate the time lag. The resulting fitted residuals are displayed in Fig. 11, and the measured time lags are listed in Table 2 along with the reduced $\chi^{2}$ of the fits. Our adopted approach is simple, but we think it is adequate for purposes of data modeling.

\section{Disk truncation and the matter propagation}

We did a detailed X-ray and UV power spectral analysis of SS Cyg during quiescence and outburst, together with the power spectral analysis of the X-ray and UV data of four other DN systems. We investigated the red noise structure resulting from the flicker noise in the accretion disks using a propogating fluctuations model and fitted the PDS, thus yielding the break frequencies where this noise starts to subside. This is a strong indication that the optically thick Keplerian flow is truncated at some large radii during the quiescent state of DNe. Similar conclusions were recently reached by Revnivtsev et al. (2012) via analysis of fast optical variability of SS Cyg in quiescence and in outburst.

We calculated a range of disk truncation radii $(10-0.3) \times 10^{9} \mathrm{~cm}$ with the WD masses $0.4-1.3 M_{\odot}$. These results are consistent with the theoretical calculations from the irradiation of WDs (King 1997), and the disk evaporation and formation of coronal flows (Meyer \& MeyerHofmeister 1994; Liu et al. 1997; Mineshige et al. 1998; de Kool \& Wickramasinghe 1999). WD irradiation yields a truncation radius about $(1-5) R_{\mathrm{WD}}$; the disk evaporation
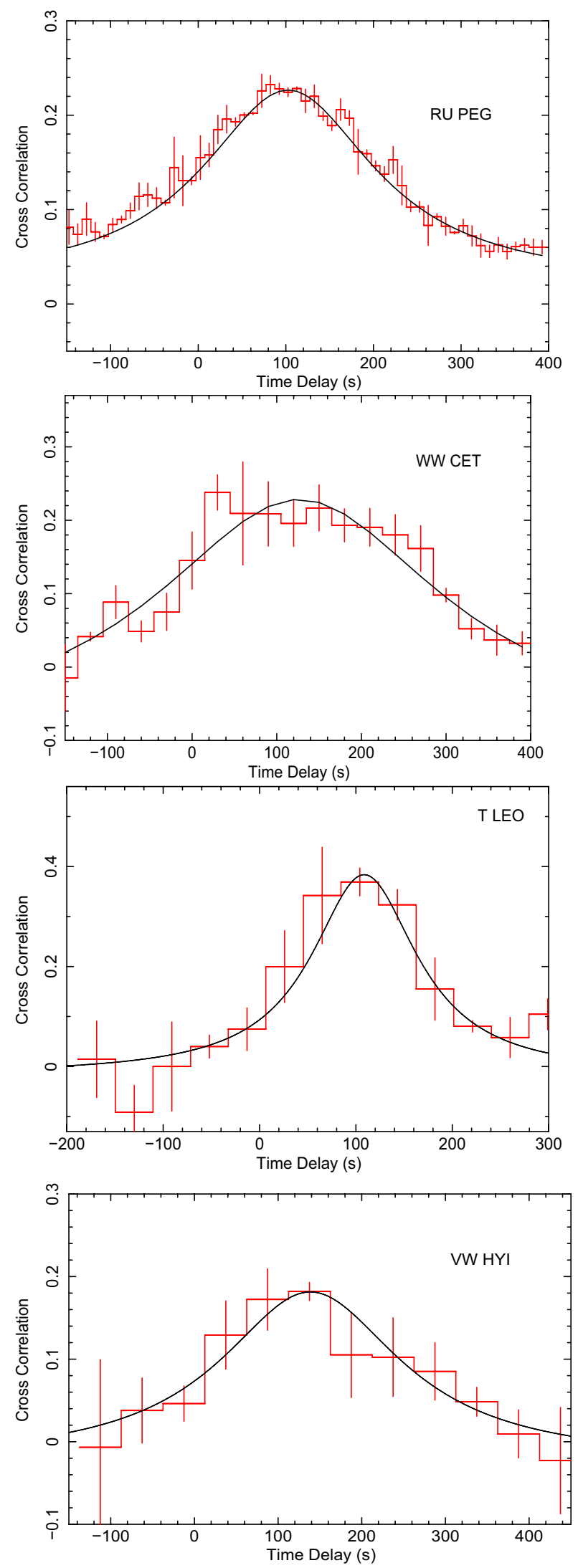

Fig. 11. Subtracted cross-correlation of the EPIC pn (X-ray) and OM (UV) light curves (see the text for details). The residuals of the fits to the cross-correlations are displayed for RU Peg, WW Cet, T Leo, and VW Hyi from the top to the bottom of the figure. Single Lorenzian fits applied are shown as solid black lines (SS Cyg has been excluded since only a single Lorentzian was already used). The reduced $\chi^{2}$ values of the fits are given in Table 2. 
and formation of coronal flows predict inner disk radii in a range $2.0-6.0 \times 10^{9} \mathrm{~cm}$. These ranges are consistent and lie within the errors of all the disk truncation radii we calculated in this work.

A general picture of the accretion flow around a WD in quiescence thus might be somewhat similar to that of the black hole/neutron star accretors with an optically thick cold outer accretion disk and an optically thin hot inner corona (see e.g. Esin et al. 1997). The appearance of hot corona in the innermost regions of the flow may significantly differ from that of ordinary rotating Keplerian disk because it is no longer fully supported by rotation, but might have a significant radial velocity component. In this case, the region where matter settles to the WD surface might differ from a simple accretion belt, anticipated by, e.g., Kippenhahn \& Thomas (1978). The radial motion of the matter in the coronal flow above the disk (close to the truncation radius) might lead to an observational appearance of single broadly peaked emission lines in eclipsed systems, discussed, e.g., by Williams (1989). Optical emission lines might also originate not in the innermost regions of the flow, see e.g. Groot et al. (2001). However, the existence of accretion columns on the WD surfaces in the case of the studied DNe in this paper is questionable because of the absence of any detectable pulsed X-ray emission. Moreover, it has been observed that the eclipsing DN exhibiting double-peaked emission lines shows evidence of extended emission during the eclipse with plasma temperatures that are different than the persistent emission, e.g., Ramsay et al. (2001).

The only DN, for which we could trace the X-ray PDS evolution through several outbursts with statistically good data was SS Cyg. We detect that the disk moves in from $(6-5) \times 10^{9} \mathrm{~cm}$ to about $1.1 \times 10^{9} \mathrm{~cm}$ from the quiescence to the peak of the optical outburst. After that it eventually moves out as the X-rays rise and go back to quiescence. Note also similar results from fast optical timing study of SS Cyg by Revnivtsev et al. (2012). This is a strong indication of the disk truncation scenario and its applicability to the explanation of the UV/X-ray delays during the DN outbursts.

The Suzaku observations of SS Cyg during the outburst stage support the existence of a disk corona as calculated from the reflection off of the disk using particularly the $6.4 \mathrm{keV}$ iron fluorescence line (Ishida et al. 2009). The authors find that the coronal region is at $r<7 \times 10^{9} \mathrm{~cm}$, which is consistent with the disk truncation radii we calculate in this work. The Suzaku data obtained in quiescence may also be consistent with a coronal structure (Ishida 2011, priv. comm.). The combined FUSE and HST data analysis of SS Cyg (in the UV wavelengths) also reveals that almost all the lines are in emission possibly from an optically thin region in the disk and/or corona, making it difficult to assess rotational broadening and chemical abundances based on absorption lines, and the data are not consistent with the simple standard disk models (Sion et al. 2010).

We checked the correlation between the variability of the $\mathrm{X}$-ray and the UV data using XMM-Newton EPIC pn and OM. We found two components in four systems; one delayed and the other undelayed (at least down to the time resolution of the data used). The only peculiarity in this respect is the case of SS Cyg (the fifth source), which only shows a delayed component. The significant variability correlation at $\Delta t \sim 0$ lag is expected to be caused by the reprocessing of X-rays (i.e., irradiation by X-rays) in the accretion disk. Such time lags are on the order of milliseconds and proportional to the light travel time, which is beyond the time resolution in our UV light curves. The delayed component detected in the five systems with 96-181 s lag is much longer than the light travel effects. In the frame work of the model of propagating fluctuations, this time lag should appear to be due to the finite time needed for matter to travel from the innermost parts of the accretion disk (UV emitting region) to the surface of the WD, where the majority of the X-ray emission is created.

A similar delay of X-rays with respect to the variations in the optical flux was detected in the magnetic accreting WD EX Hya (Revnivtsev et al. 2011). In this case, it is known that the accretion disk is truncated due by the interaction with the WD magnetosphere. The time lag detected in this system is around seven seconds (Revnivtsev et al. 2011), while the break frequency is $\sim(1.7 \pm 0.2) \times 10^{-2} \mathrm{~Hz}$.

The ratio of these two time scales defined by the break in the power spectrum $t_{\text {break }}$ and the time lag $t_{\text {lag }}$ - is significantly different between the case of magnetic accreting WD, EX Hya, and the case of nonmagnetic, DNe. This is expected in the framework of the model of propagating fluctuations.

As shown in Revnivtsev et al. (2009), $t_{\text {break }}$ is close to the time of rotation of matter on the Keplerian orbit at the inner edge of the disk. The travel time ( $\approx$ time lag) in the case of magnetic intermediate polar systems is approximately equal to the time of free fall from the inner boundary of the disk to the WD surface. Matter moves along the magnetic field lines in the WD magnetosphere.

In the case of $\mathrm{DNe}$ that are nonmagnetic systems, this time scale is a time of viscous propagation of matter fluctuations from the inner radius of the optically thick accretion disk to the region where the bulk of the X-ray generation occurs, close to the WD surface (Mukai et al. 1997; Nucita et al. 2009). We should emphasize here that, below the truncation radius, the accretion flow is optically thin and thus hot, therefore its viscous properties are different from those of the outer non-active disk.

If we assume that the innermost parts of the accretion flow are optically thin and virialized, then the radial velocity $v_{\mathrm{r}}$ in this inner part of the flow is $v_{\mathrm{r}} \sim \alpha v_{\mathrm{K}}$, where $v_{\mathrm{K}}$ is the Keplerian velocity, while in the case of a simple freefall, the radial velocity can be estimated as $v_{\mathrm{r}} \sim v_{\mathrm{K}}$. The ratio of $t_{\text {break }} / t_{\mathrm{lag}}$ in these two cases (magnetized and nonmagnetized CVs) should depend on $\alpha$. The ratio of $t_{\text {break }} / t_{\text {lag }}$ approximately equals (as expected) $\sim 2 \pi$ in the case of a magnetic CV EX Hya (Revnivtsev et al. 2011), and it is approximately four times smaller in the case of the nonmagnetic DN systems, yielding a value of $\alpha \sim 0.25$ for the hot inner part of the accretion flow. We stress that this estimate should be treated with caution because of our very simplified approach. Our viscosity estimate significantly depends on our conclusions that the disk is truncated at large distances (>1-5 $R_{\mathrm{WD}}$ ) from the WD surface. In some previous studies where the matter travel distance was assumed to be much smaller than we deduce from our analysis, authors obtained much lower values of $\alpha$ in the inner accretion flow where they presumed a standard optically thick disk reaching to the WD with a narrow boundary layer bright and optically thin in the X-rays (e.g. Godon \& Sion 2005).

\section{Summary and conclusions}

We have presented the power spectral analysis of five DNe systems in quiescence and searched for cross-correlations between the X-ray and UV light curves. We studied the red noise structure resulting from the flicker noise in the accretion disks and modeled the PDS, yielding the break frequencies. This is a strong indication that the optically thick Keplerian flow is truncated at some large radii during the quiescent state of $\mathrm{DNe}$ and coronal flows are formed (optically thick-thin disk transition). These 
structures may be extended on the accretion disk and be emitting at low levels that require high sensitivity for detection. Such structures may be created by the disk evaporation, as suggested earlier. Our range of break frequencies $(1-6 \mathrm{mHz})$ yield a range of radii $(10-0.3) \times 10^{9} \mathrm{~cm}$ for the inner disk radius with the WD masses $0.4-1.3 M_{\odot}$.

In addition, we also analyzed the X-ray outburst data of SS Cyg taken at different epochs and derived that the disk moves in from the large truncation radius during quiescence towards the WD during the optical peak of the outburst and recedes as the $\mathrm{X}$-rays peak in the outburst, finally to the quiescent inner disk radius. Our findings are consistent with the previous suggestions of an accretion disk corona existing in SS Cyg.

We modeled the cross-correlations of the quiescent UV and $\mathrm{X}$-ray light curves of our sample of DNe to yield time delays of $\mathrm{X}$-rays in the range 96-181 s, which indicates the time lag of emission as the matter travels from the innermost parts of a truncated accretion disk (UV emitting region) to the surface of the WD, where the majority of the X-ray emission is created (i.e., the X-ray photons are delayed). In four of the systems (except for SS Cyg), we also detect zero time-lag correlation indicating the existence of irradiation and reprocessing of X-rays from the cold disk consistent with the light-crossing time scales of the systems.

Finally, in the framework of the propagating fluctuations model we used the ratio of the break time scale and the time lag of magnetic CV EX Hya and DN systems to derive an estimate of 0.25 for the $\alpha$ parameter in the inner (optically thin) parts of the accretion flow of DNe disks.

Acknowledgements. S.B. acknowledges financial support from both the TUBITAK National Observatory (TUG) and the Space Research Institute of Russian Academy of Sciences for her visits to Space Research Institute (IKI) in 2010-2011. M.R. acknowledges the support by the grants of President of Russian Federation MD-1832.2011.2, RFBR 10-02-00492, program P21 of the Presidium of the Russian Academy of Sciences/RAS, program OFN17 of the Division of Physical Sciences of the RAS and Dynasty Foundation.

\section{References}

Arévalo, P., \& Uttley, P. 2006, MNRAS, 367, 801

Balman, Ş., Godon, P., Sion, E. M., et al. 2011, ApJ, 741, 84

Baptista, R., \& Bortoletto, A. 2004, AJ, 128, 411

Bradt, H. V., Rothschild, R. E., \& Swank, J. H. 1993, A\&AS, 97, 355

Bruch, A. 1992, A\&A, 266, 237

Bruch, A. 2000, A\&A, 359, 998

Churazov, E., Gilfanov, M., \& Revnivtsev, M. 2001, MNRAS, 321, 759

de Kool, M., \& Wickramasinghe, D. 1999, MNRAS, 307, 449

Done, C., Gierliński, M., \& Kubota, A. 2007, A\&ARv, 15, 1

Esin, A. A., McClintock, J. E., \& Narayan, R. 1997, ApJ, 489, 865
Gilfanov, M., \& Arefiev, V. 2005, unpublished [arXiv: astro-ph/0501215] Giovannelli, F., \& Sabau-Graziati, L. 1999, MmSAI, 70, 987

Godon, P., \& Sion, E. M. 2005, MNRAS, 361, 809

Godon, P., Regev, O., \& Shaviv, G. 1995, MNRAS, 275, 1093

Groot, P. J., Rutten, R. G. M., \& van Paradijs, J. 2001, A\&A, 368, 183

Horne, K. 1985, MNRAS, 213, 129

Ishida, M., Okada, S., Hayashi, T., et al. 2009, PASJ, 61, 77

Jansen, F., Lumb, D., Altieri, B., et al. 2001, A\&A, 365, L1

Jahoda, K., Markwardt, C. B., Radeva, Y., et al. 2006, ApJS, 163, 401

King, A. R. 1997, MNRAS, 288, L16

Kippenhahn, R., \& Thomas, H.-C. 1978, A\&A, 63, 265

Kuulkers, E., Norton, A., Schwope, A., \& Warner, B. 2006, in Compact stellar X-ray sources, eds. W. Lewin, \& M. van der Klis (Cambridge, UK: Cambridge University Press), Cambridge Astrophysics Series, 39

Kuulkers, E., Henden, A. A., Honeycutt, R. K., et al. 2011, A\&A, 528, A152

Lasota, J.-P. 2001, New Astron. Rev., 45, 449

Lasota, J.-P. 2004, RMxAC, 20, 124

Linnell, A. P., Szkody, P., Gänsicke, B., et al. 2005, ApJ, 624, 923

Liu, B. F., Meyer, F., \& Meyer-Hofmeister, E. 1997, A\&A, 328, 247

Lombardi, R., Gaudenzi, S., \& Giovannelli, F. 1987, Ap\&SS, 130, 275

Lynden-Bell, D., \& Pringle, J. E. 1974, MNRAS, 168, 603

Lyubarskii, Y. E. 1997, MNRAS, 292, 679

Mason, K. O., Breeveld, A., Much, R., et al. 2001, A\&A, 365, L36

McGowan, K. E., Priedhorsky, W. C., \& Trudolyubov, S. P. 2004, ApJ, 601, 1100

Meyer, F., \& Meyer-Hofmeister, E. 1994, A\&A, 288, 175

Mineshige, S., Liu, B., Meyer, F., \& Meyer-Hofmeister, E. 1998, PASJ, 50, L5

Miyamoto, S., Kimura, K., Kitamoto, S., Dotani, T., \& Ebisawa, K. 1991, ApJ, 383,784

Mukai, K., Wood, J. H., Naylor, T., Schlegel, E. M., \& Swank, J. H. 1997, ApJ, 475,812

Narayan, R., \& Popham, R. 1993, Nature, 362, 820

Nucita, A. A., Maiolo, B. M. T., Carpano, S., et al. 2009, A\&A, 504, 973

Pandel, D., Córdova, F. A., \& Howell, S. B. 2003, MNRAS, 346, 1231

Pandel, D., Córdova, F. A., Mason, K. O., \& Priedhorsky, W. C. 2005, ApJ, 626, 396

Patterson, J., \& Raymond, J. C. 1985, ApJ, 292, 550

Perna, R., McDowell, J., Menou, K., Raymond, J., \& Medvedev, M. V. 2003, ApJ, 598, 545

Ramsay, G., Poole, T., Mason, K., et al. 2001, A\&A, 365, L288

Rana, V. R., Singh, K. P., Schlegel, E. M., \& Barrett P. E. 2006, AdSpR, 38, 2847

Revnivtsev, M., Churazov, E., Postnov, K., \& Tsygankov, S. 2009, A\&A, 507, 1211

Revnivtsev, M., Burenin, R., Bikmaev, I., et al. 2010, A\&A, 513, A63

Revnivtsev, M., Potter, S., Kniazev, A., et al. 2011, MNRAS, 411, 1317

Revnivtsev, M., Burenin, R. A., Tkachenko, A., Yu., et al. 2012, Astron. Lett., 38,271

Scaringi, S., Körding, E., Uttley, P., et al. 2012, MNRAS, 421, 2854

Sion, E. M., Godon, P., Myzcka, J., \& Blair, W. P. 2010, ApJ, 716, L157

Strüder, L., Briel, U., Dennerl, K., et al. 2001, A\&A, 365, L18

Turner, M. J. L., et al. 2001, A\&A, 365, L27

Uttley, P., \& McHardy, I. M. 2001, MNRAS, 323, L26

Uttley, P., Wilkinson, T., Cassatella, P., et al. 2011, MNRAS, 414, L60

Warner, B. 1995, Cataclysmic Variable Stars (Cambridge: Cambridge University Press)

Warner, B., \& Nather, R. E. 1971, MNRAS, 152, 219

Williams, R. E. 1989, AJ, 97, 1752 